\title{
Structure and functional impact of seed region variant in MIR-499 gene family in bronchial asthma
}

\author{
Eman A. Toraih ${ }^{1 *}$, Mohammad H. Hussein ${ }^{2}$, Essam Al Ageeli ${ }^{3}$, Eman Riad ${ }^{4}$, Nouran B. AbdAllah ${ }^{5}$, Ghada M. Helal ${ }^{6}$ \\ and Manal S. Fawzy ${ }^{7,8^{*}}$
}

\begin{abstract}
Background: Small non-coding RNAs (microRNAs) have been evolved to master numerous cellular processes. Genetic variants within microRNA seed region might influence microRNA biogenesis and function. The study aimed at determining the role of microRNA-499 (MIR-499) gene family polymorphism as a marker for susceptibility and progression of bronchial asthma and to analyze the structural and functional impact of rs3746444 within the seed region.

Methods: Genotyping for 192 participants (96 patients and 96 controls) in the discovery phase and 319 subjects (115 patients and 204 controls) in the replication phase was performed via Real Time-Polymerase Chain Reaction technology. Patients underwent the methacholine challenge test and biochemical analysis. Gene structural and functional analysis, target prediction, annotation clustering, and pathway enrichment analysis were executed. Predicted functional effect of rs37464443 SNP was analyzed.

Results: miR-499 gene family is highly implicated in inflammation-related signaling pathways. Rs374644 (A > G) in MIR499A and MIR499B within the seed region could disrupt target genes and create new genes. The $G$ variant was associated with high risk of developing asthma under all genetic association models ( $G$ versus A: $O R=3.27,95 \%$ $\mathrm{Cl}=2.53-4.22 ; \mathrm{GG}$ versus $\mathrm{AA}: \mathrm{OR}=9.52,95 \% \mathrm{Cl}=5.61-16.5 ; \mathrm{AG}$ versus $\mathrm{AA}: \mathrm{OR}=2.13,95 \% \mathrm{Cl}=1.24-3.46 ; \mathrm{GG}+\mathrm{AG}$ versus $\mathrm{AA}: \mathrm{OR}=4.43,95 \% \mathrm{Cl}=2.88-6.82)$. GG genotype was associated with poor pre-bronchodilator $\mathrm{FEV}_{1}(p=0$. 047) and the worst bronchodilator response after Salbutamol inhalation, represented in low peaked expiratory flow rate $(p=0.035)$.
\end{abstract}

Conclusions: miR-499 rs3746444 (A > G) polymorphism was associated with asthma susceptibility and bronchodilator response in Egyptian children and adolescents. Further functional analysis is warranted to develop more specific theranostic agents for selecting targeted therapy.

Keywords: Asthma, Egyptians, miR-499a, miR-499b, Polymorphism, Airway hyper-responsiveness, qRT-PCR

\section{Background}

Bronchial asthma is a chronic heterogeneous respiratory disease that is characterized by airway inflammation, recurring bronchial obstruction, and airway hyperresponsiveness [1, 2]. Most common histopathological features are inflammatory cell infiltration, sub-basement

\footnotetext{
*Correspondence: emantoraih@gmail.com; manal2_khashana@ymail.com

${ }^{1}$ Genetics Unit, Department of Histology and Cell Biology, Faculty of

Medicine, Suez Canal University, Ismailia P.O. 41522, Egypt

${ }^{7}$ Department of Medical Biochemistry, Faculty of Medicine, Suez Canal

University, Ismailia P.O. 41522, Egypt

Full list of author information is available at the end of the article
}

fibrosis, smooth muscle hypertrophy, mucus hypersecretion, injury to epithelial cells, and angiogenesis [3, 4]. Treatment with anti-inflammatory drugs or bronchodilators usually improves some of these features. Nevertheless, therapeutic response relies on the interplay between environmental exposure and genetic background [2] Despite several advances over the past decades in understanding the underlying mechanisms involved in the disease, there are no current satisfactory strategies for the cure or prevention of long-term decline in pulmonary function [5]. Given the significant morbidity and 
burden of childhood asthma worldwide, better therapeutic modalities are mandatory to counteract the development and progression of the disease. In prior studies, a sizeable proportion of genetic influence existed, ranging from 35 to $95 \%$ for asthma and 30 to $66 \%$ for bronchial hyper-responsiveness [6]. Genome-wide association studies provide evidence for multiple novel loci associated with the disease. However, the exact maestro fine tuning these putative genes is still uncovered.

In early 90's, the presence of small non-coding RNAs (ncRNAs) was discovered in the mammalian genome [7]. These microRNAs are transcriped via specific cellular machinery to form short single-stranded mature RNAs of 19-24 nucleotides long. They function by complementary base pairing with mRNA targets, leading to its degradation or translational repression [8]. They are estimated to modulate gene expression of $60 \%$ of protein-coding genes, and to regulate many cellular processes; including proliferation, apoptosis, immunomodulation, stress response, and angiogenesis [9]. As a result the focus of human genome studies has witnessed a shift from mRNAs to ncRNAs as major key players in human disorders. Currently, there are emerging opportunities for targeting these disruptions of ncRNAs using novel therapeutic approaches. Some strategies aimed to increase the levels of abnormally down-regulated microRNAs via epigenetic drugs as DNA demethylating agents and histone deacetylase (HDAC) inhibitors or by replacement of miRNAs using virus delivery systems [10]. On the other hand, over-expressed microRNAs in human diseases can be sequestered by anti-miRNA oligonucleotides, miRNA sponges, miRNA masking and small molecule inhibitors [8].

In silico analysis and surveying the literature revealed the deregulation of microRNAs in various pulmonary diseases (Fig. 1). Several lines of evidence suggest a key role for hsa-microRNA-499a (miR-499a) in modulating the immune response, cell proliferation, apoptosis, neuromuscular regulation and neoangiogenesis [11, 12]. Exploring gene targets of miR-499a by computational tools identified inflammatory-related gene targets, including IL-13 and Il-23, which represent important mediators in asthma KEGG pathway [ID hsa05310] (microRNA.org). A single nucleotide polymorphism (SNP), rs3746444 (A > G), is located in the seed sequence of miR-499a-3p, a region essential for miRNAmediated silencing mechanism. SNPs within mRNAbinding site of miRNAs may influence mRNA gene set, target binding ability, or pre-miRNA maturation process, which in turn, could alter the susceptibility to develop human diseases. The rs3746444 MIR-499a SNP was found to be associated with higher risk of rheumatoid arthritis [12, 13], coronary artery disease [14], Behcet's disease [15], and ankylosing Spondylitis [16]. Hence, the current study was conducted to investigate the association between rs3746444 polymorphism and susceptibility to asthma disease in children and adolescents, and to further assess computationally its impact on the clinical outcome and bronchial hyper-responsiveness (BHR) in a group of Egyptian asthmatic children and adolescents.

\section{Methods}

\section{Structural gene analysis}

Genetic analysis of MIR499A gene was performed using Genecards.org, Ensembl.org, and NCBI. DNA and RNA sequences were retrieved from miRBase.org. Multiple sequence alignment and phylogenetic tree construction were implemented to identify similarity regions in different species by Ensembl.org and with its cluster gene MIR499B by Rcoffee v11.0, a specific method for noncoding RNA [17]. Analysis by PolymiRTS Database v3.0 (Polymorphism in microRNAs and their TargetSites) was conducted to predict SNPs and INDELs in the whole gene (http://compbio.uthsc.edu/miRSNP/home.php) [18]. Variant calls in the chromosomal region were obtained from Ensembl.org and UCSC via usegalaxy.org platform.

\section{In silico target gene prediction}

Next, list of computationally predicted and experimentally validated gene targets (Additional file 1: Table S2) were retrieved from multiple databases including DIANA-microT-CDS v5.0 (http://diana.imis.athenainnovation.gr/DianaTools/index.php?r=microT_CDS/index), miRBase (http://www.mirbase.org/), TargetScanHuman v6.2 (http://www.targetscan.org/), miRDB (http://mirdb.org /miRDB/), miRNAMap v2.0 (http://mirnamap.mbc.nctu. edu.tw/), and DIANA-TarBase v7.0 algorithm (http://diana. imis.athena-innovation.gr/DianaTools/index.php? $r=$ tarbase/ index) databases. Result intersection and statistical validation were performed as described previously [12].

\section{Functional annotation clustering and pathway enrichment analysis}

Functional analysis of the gene list was done via DianamiRPath v3.0 software for gene ontology (GO) terms and Kyoto encyclopedia of genes and genomes (KEGG) pathways [19]. Fisher's exact test/hypergeometric statistical test was applied at microT-CDS threshold of 0.8 and $P$ value threshold at 0.05 . Gene Ontology (GO) system of classification was carried out to interpret the target gene sets of miR-499a-3p, miR-499a-5p, miR$499 \mathrm{~b}-3 \mathrm{p}$, and miR-499b-5p based on their functional characteristics. The attributes of targets were classified and ranked in the context of biological process, molecular function, or cellular component. Further comparison of functional categories between miR-499a and miR499b targets was conducted by miRpair2Go webplatform using two combined miRNA target prediction 


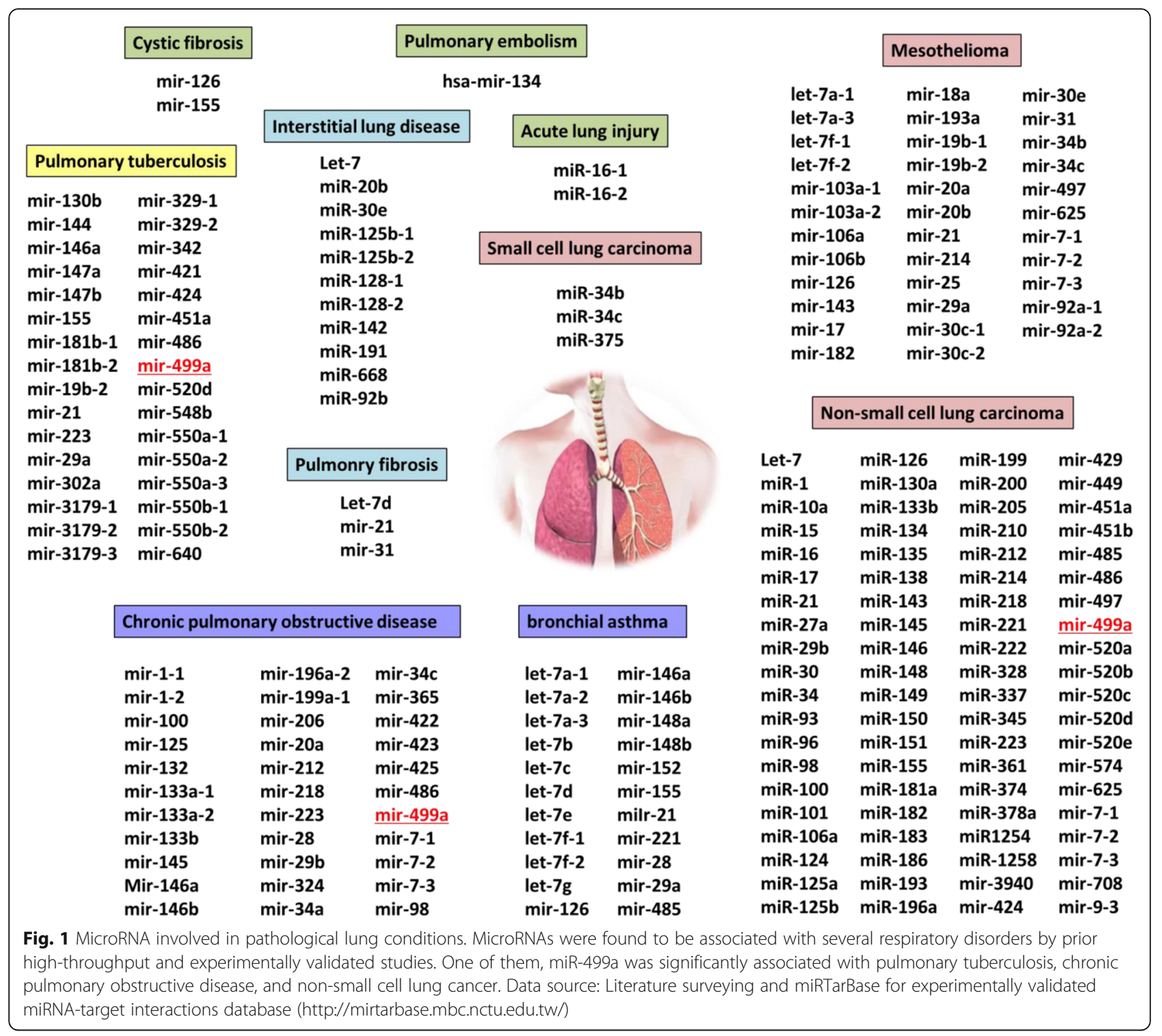

methods (TargetScan and miRanda) and moderate hierarchial filtering level (http://compbio.uthsc.edu/miR2GO/ help.php) [20].

\section{Predicted functional effect of rs37464443 SNP}

Distribution of SNPs in miR-499 was analyzed via miRdSNP database (http://mirdsnp.ccr.buffalo.edu/): a database of disease-associated SNPs to identify the spatial relationship of the miRNA with target sites on the 3'UTR of human genes and to further explore the molecular mechanism of gene deregulation at the posttranscriptional level [21]. The predicted functional impact of miR-499 rs3746444 variant was performed using miRmut2Go (http://compbio.uthsc.edu/miR2GO) to analyze the changes of target genes caused by miRNA mutations and view the functional impacts of these changes in the context of comparative functional GO analysis using the same filters described in our prior work [9]. Advanced gene set enrichment analysis was employed using the Gene Trail program with multiple testing correction via false discovery rate (FDR) estimation and significant level at 0.05 (https://genetrail2. bioinf.uni-sb.de/) [22]. In addition, mfold RNAfold (http://unafold.rna.albany.edu/?q=mfold) and KineFold web-servers (http://kinefold.curie.fr/) were conducted to predict the secondary structures of RNA sequence in $A$ and $G$ alleles.

\section{Study participants}

A total of 211 asthmatic patients and 300 controls (age range 3 to 18 years old) were enrolled in the study. In the discovery stage: the study participants were composed of 96 patients and 96 controls. Patients were obtained from the Pediatrics outpatient clinic of Suez 
Canal University Hospital (SCUH), Ismailia, and Chest and tuberculosis Department, Kasr Al-Ainy Hospital, Giza. Whereas, in the replication phase: other independent cohorts of 115 patients and 204 controls were recruited from SCUH, Ismailia. They were diagnosed and assessed according to the Global Initiative for Asthma (GINA) guidelines [23]. A thorough clinical assessment was performed for determining disease severity, therapeutic history, and co-morbidities as previously described [2]. Controls had no history of wheezes, atopy, or any other respiratory diseases. Chest X-ray was done for participants to exclude concurrent chest disease. Body mass index (BMI) percentile of patients and controls was estimated and adjusted for age and sex using an online pediatric calculator (http://www.quesgen.com/ BMIPedsCalc.php) [24]. Sexual maturity rating was determined based on Tanner classification [25]. The study was conducted in accordance with the guidelines in the Declaration of Helsinki and had the approval of the Ethics Committee of Faculty of Medicine, Suez Canal University. Informed consent was obtained from participants' parents.

\section{Spirometry and methacholine challenge test}

Baseline pulmonary function test assessment was done using an electronic Spirometer (BTL-08 Spiro Pro system; BTL) with a valve-spacer device following the guidelines of the American Thoracic Society/European Respiratory Society (ATS/ERS) [26]. Baseline lung parameters were documented [27]. Repeated postbronchodilator forced Spirometry was performed $15 \mathrm{~min}$ after administering a $400 \mu \mathrm{g}$ dose of inhaled Salbutamol (Ventolin; GlaxoSmithKline). BDRBASE, change in $\mathrm{FEV}_{1}$ as a percent of baseline forced expiratory volume at $1 \mathrm{~s}$ $\left(\mathrm{FEV}_{1}\right)$, was calculated with the following equation [= ((postbronchodilator $\mathrm{FEV}_{1}$ - prebronchodilator $\mathrm{FEV}_{1}$ ) / prebronchodilator $\left.\mathrm{FEV}_{1}\right) \times 100$ ] [27]. Additionally, bronchoconstriction provocation via methacholine challenge test (MCT) was done to assess bronchial hyperresponsiveness. Methacholine solution, mixed with saline with the following gradient doses $(0.06,0.125,0.25$, $1,2,4,8,16 \mathrm{mg} / \mathrm{ml}$ ), were aerosolized using a nebulizer attached to an air compressor at 5 min interval. Progressive increase in concentration was used, until the patient encountered a significant worsening in lung function, with a drop in FEV1 of $20 \%$ or more. BHR was categorized according to the American Thoracic Society guidelines with positive cutoff value defined as a PC20 below $8 \mathrm{mg} / \mathrm{ml}[28,29]$.

\section{Biochemical analysis}

Blood samples were collected in EDTA Vacutainers. Absolute eosinophil count (AEC) was calculated using the Coulter Counter. Total IgE was measured by enzyme- linked immunosorbant assay (ELISA). Absolute esinophilic count $<0.1 \times 10^{3} / \mu \mathrm{l}$ and total IgE concentrations $<90 \mathrm{IU} / \mathrm{ml}$ were defined as normal [30].

\section{Genotyping of seed region variant}

Genomic DNA was purified from whole blood using QIAamp DNA Blood Mini kit (Catalog No. 51104; Qiagen) following the manufacturer's protocol. Extracted DNA purity and concentration were assessed by NanoDrop ND-1000 (NanoDrop Technologies, Inc. Wilmington, DE, USA). Genotyping for the hsa-miR-499a (rs3746444) was assayed using Real-Time polymerase chain reaction (RT-PCR) allelic discrimination technology. PCR reactions were run blindly in duplicates in a $25-\mu \mathrm{l}$ final volume containing 20 ng genomic DNA, TaqMan Universal PCR Master Mix, No UNG (4440043), and TaqMan SNP Genotyping Assay Mix (assay ID C_2142612_30, Applied Biosystems) with $100 \%$ concordance rate for genotype calls. Appropriate controls were used in each reaction. PCR amplification was done using StepOne ${ }^{\text {tm }}$ Real-Time PCR System (Applied Biosystems, USA) [12]. Allelic discrimination was called by the SDS software version 1.3.1 (Applied Biosystems).

\section{Statistical analysis}

Statistical analysis was performed using PCORD v.5.0, $\mathrm{R}$ programming language and the "Statistical Package for the Social Sciences (SPSS) for windows" software, version 22. Allele and genotype frequencies and carriage rate were calculated as previously described [31]. The Hardy-Weinberg equilibrium was estimated using the Online Encyclopedia for Genetic Epidemiology (OEGE) software (http:// www.oege.org/software/hwe-mr-calc.shtml) and tested for goodness of fit by chi square test. Genotype and allele frequencies were compared between asthmatic patients and control subjects using the chi-square test. Adjusted odds ratios (OR) with a $95 \%$ confidence interval $(\mathrm{CI})$ by logistic regression analysis were calculated for multiple genetic association models. Data distribution was checked by the Kolmogorov-Smirnov test. Appropriate data presentation and test were used for comparison between groups. A twotailed $P$-value of 0.05 was considered statistically significant. Both ordination and two-way agglomerative hierarchal clustering techniques were applied to the data for multivariate analysis [32].

\section{Results}

Structural gene analysis and comparative genomics Human miR-499a gene (MIR499A; ENSG 00000207635) is located along the long arm of chromosome 20q11.22 spanning 122 bp long (Genomic coordinates at 20:34,990,376-34,990,497 on the forward strand; according to the Human Genome Assembly GRCh38, release annotation 108). MIR499A gene exists within intron 19 of 
myosin, heavy chain $7 \mathrm{~B}$, cardiac muscle, beta $M Y H 7 B$ gene and overlapping MIR499B gene (ENSG00000283441; 73 bp in length; 20: 34,990,400-34,990,472 on the reverse strand) (Fig. 2). Similar orthologs are present in other species; mouse MIR-499 was also mapped to intron 19 of the Myh7b gene on chromosome 2 (Mus musculus;
2:155,622,880-155,622,958 (+); GRCm38). Whereas rat MIR-499, existed on chromosome 3 (Rattus norvegicus; 3:151,138,862-151,138,926 (+); Rnor6.0). Multiple sequence alignment showed MIR499A gene to display a high level of conservation throughout 17 mammalian species (Fig. 3).

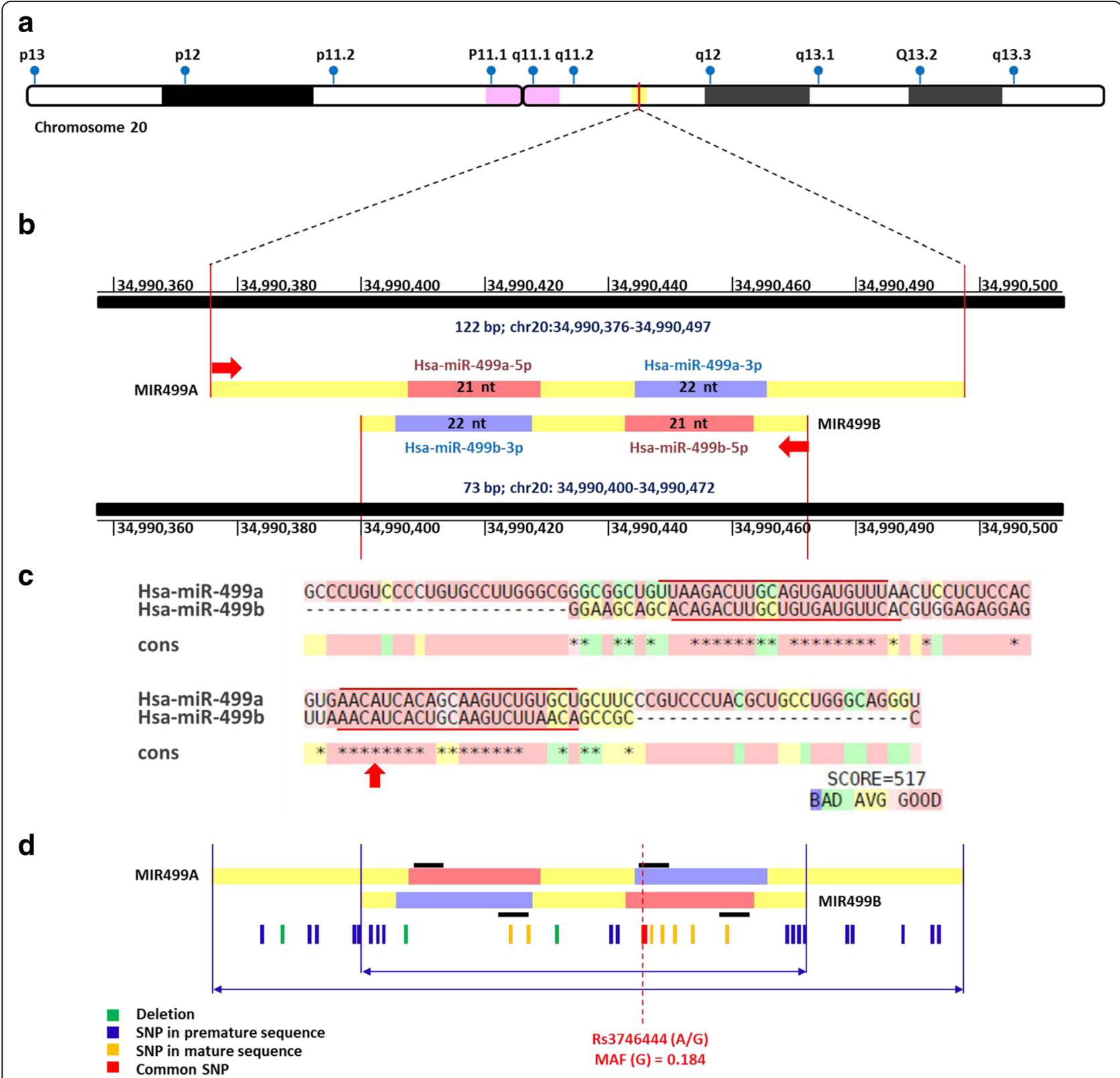

Fig. 2 Genomic structure of human MIR-499A gene. (a) Chromosomal location along the long arm of chromosome 20q11.22. (b) MIR499A gene (ENSG 00000207635) is located on the forward strand spanning 122 bp long (Genomic coordinates at 20:34,990,376-34,990,497; according to the Human Genome Assembly GRCh38, release annotation 108). Whereas, MIR499B gene (ENSG00000283441) is located on the other strand spanning 73 bp in length $(20: 34,990,400-34,990,472)$. The direction of miRNA transcription is shown by red arrows. MiR-499a is transcribed from the forward strand. MiR$499 \mathrm{~b}$ is transcribed from the reverse strand. Each miRNA has two embedded mature forms; one at the $5^{\prime}$ end (5p) in pink (21 nucleotides), the other at $3^{\prime}$ end in blue (22 nucleotides). (c) High conservation shown by the pairwise alignment produced using Rcoffee platform. Positions of mature forms are shown by the red line. The red arrow shows the studied SNP in the study (rs3746444; A/G). (d) Variant analysis of the MIR499A gene. A total of 30 variants (3 deletion and 27 SNPs), were identified. Of them, 19 variants are overlapped with MIR499B gene variants. Seed region is represented by a black bar at the $5^{\prime}$ end of each mature miRNA 


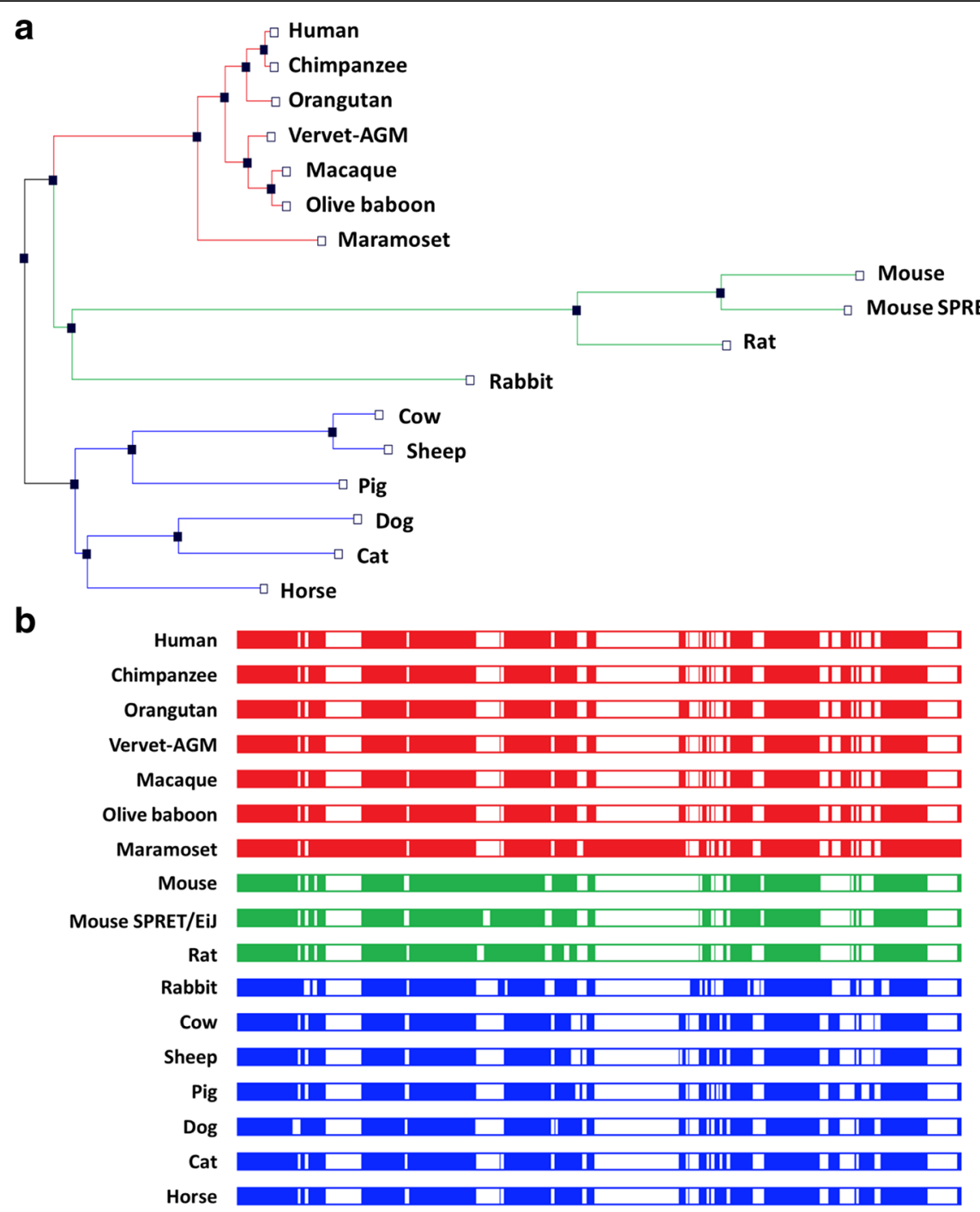

Fig. 3 Human MIR499A shows high conservation across different species. (a) Phylogenetic tree showing the degree of similarity among different species. Close similarities exist with higher primates than remote species. (b) Multiple sequence alignment was done via Ensemble.org. The figure shows that miR-499a is existed in a highly conserved genomic locus

MIR499A consists of a single exon that encodes for a single transcript (MI0003183) of 122 bp long. Whereas, its clustered MIR499B forms a shorter transcript of 73 bp long from the reverse strand. Variant analysis of MIR499A identified the presence of 30 non-coding transcript exon variants (27 SNPs and 3 deletions) in MIR499A including 19 overlapping variants (17 SNPs and 2 deletions) existed in MIR499B. All variants were rare except rs3746444 (A/G alleles) at the position 20:34,990,448 (GRCh38) with minor allele frequency (MAF) of 0.184. The rs3746444 variant exists in the seed region of miR-499a-3p; AC[A/G]UCAC.

\section{In silico target gene prediction}

Hundreds of genes were predicted to be targeted by miR-499a and miR-499b using multiple microRNA databases. Though miRNAs form secondary hairpin loop, with complementary sequences in their structure; different gene sets were predicted to be targeted by both mature forms synthesized from either arm. A total of 1890 genes was predicted to be influenced by miR-499a (919 genes by $3 p, 810$ by $5 p$, and 161 genes by both). However, miR-499b was involved in manipulating the transcription of 1528 genes (910 genes by $3 \mathrm{p}, 514$ by $5 \mathrm{p}$, and 104 genes by both forms). Due to sequence similarity identified between miR-499a and miR-499b, their mature forms shared target genes; specifically 832 common genes are targeted by both miR-499a-3p and miR-499b-3p, whereas, 93 genes were the same for both $5 p$ forms (Fig. 4a). 


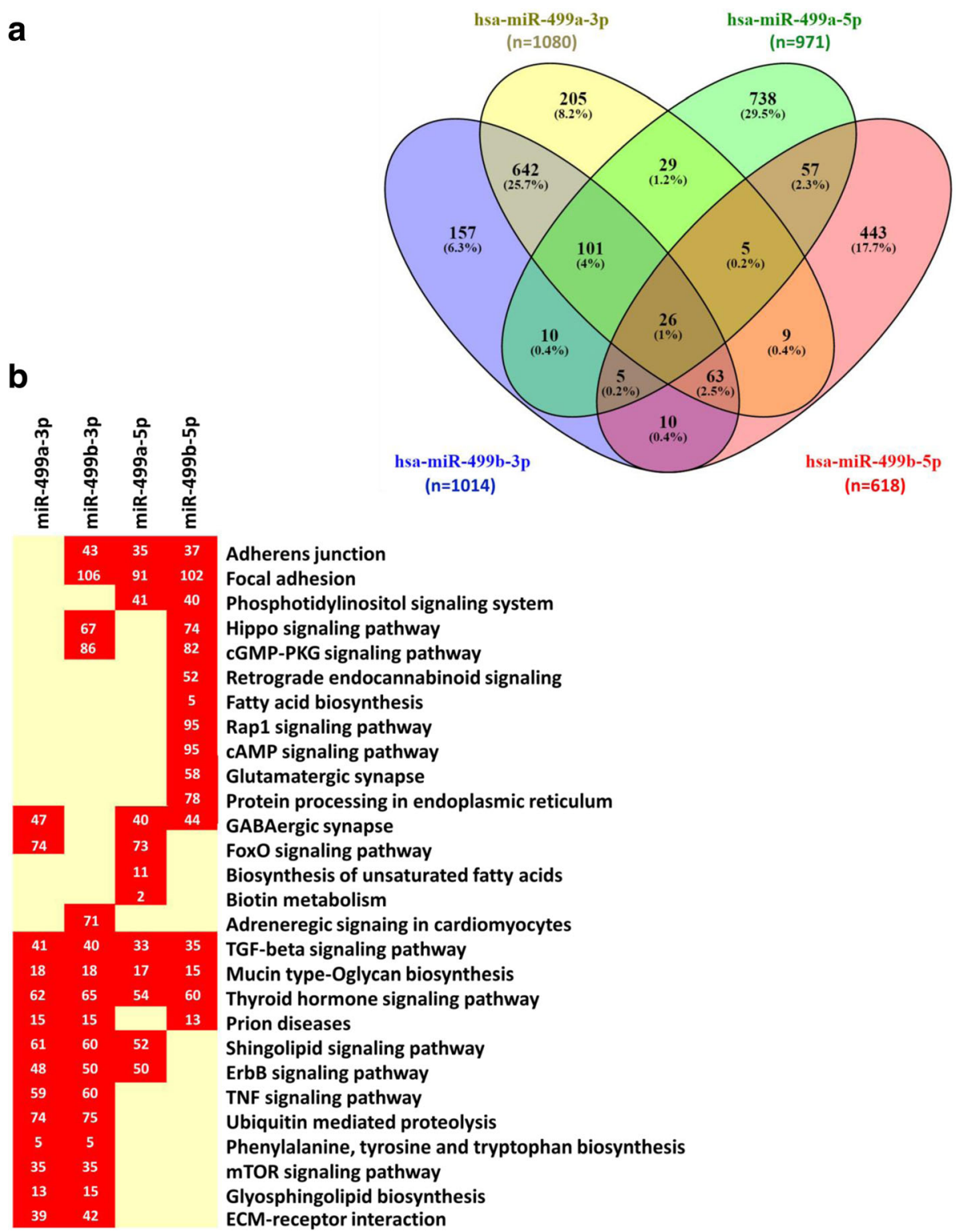

Fig. 4 Enrichment pathway analysis of MIR-499 gene cluster targets. (a) Venn diagram showing the intersection of gene targets for each microRNA. Gene targets were retrieved from microT-CDS Diana tools (http://diana.imis.athena-innovation.gr/DianaTools/index.php?r=microT_CDS/index) with threshold set at 0.7 and significance $<0.05$. Venn diagram was drawn using Venny v2.0 (http://bioinfogp.cnb.csic.es/tools/venny/). The figure shows that hundreds of gene targets are complementary to miR-499a and miR-499b. Some of these genes are commonly targeted by both genes, showing some similarities in their function. However, other gene targets that are distinct for each mature form, indicate the presence of unique function for each of them. (b) Heat map showing top significant KEGG pathways involved by miRNA targets. Result intersection and filtration after FDR correction was applied. Fisher's Exact test (hypergeometeric distribution) was used for statistical analysis. Heat map represent some of the significant KEGG pathways related to the target gene set of each mature miRNA. The number in the heat map represents the number of gene targets in each pathway affected by the corresponding miRNA. The four mature forms of MIR-499 gene family is involved in TGF-beta signaling pathway and mucin biosynthesis. Additionally, miR-499a-5p along with miR-499b are enrolled in adherence junction and focal adhesion

Functional annotation clustering and pathway enrichment analysis

KEGG pathway enrichment analysis showed enrollment of miR-499 gene targets in remodeling and inflammationrelated signaling pathways; including mucus biosynthesis and secretion, sphingolipid signaling, phosphatidylinositol signaling cell adhesion (focal adhesion and adherence junction pathways), fibrogenic and immune-modulator pathways (TGF beta signaling and TNF signaling pathways) (Fig. 4b). Functional clustering annotation of these gene sets identified the most significant their molecular activities, biological processes, pathways, and the cellular components where these 
genes execute their functions (Fig. 5). Comparison between the two microRNAs by miRpair $2 \mathrm{GO}$ explored the functional similarity scores for gene ontology to be 0.676 for biological process; 0.412 for molecular function, and 0.833 for cellular components. These targets were significantly clustered in four chromosomes; namely 67 genes on chromosome 3, 47 genes on chromosome 12, 31 genes on chromosome 16, and 30 genes on chromosome 19.

\section{Predicted functional effect of rs3746444 SNP}

In silico analysis identified the rs3746444 variant to overlap 3 genes. It lies in the intron of $M Y H 7 B$ gene

\section{a}
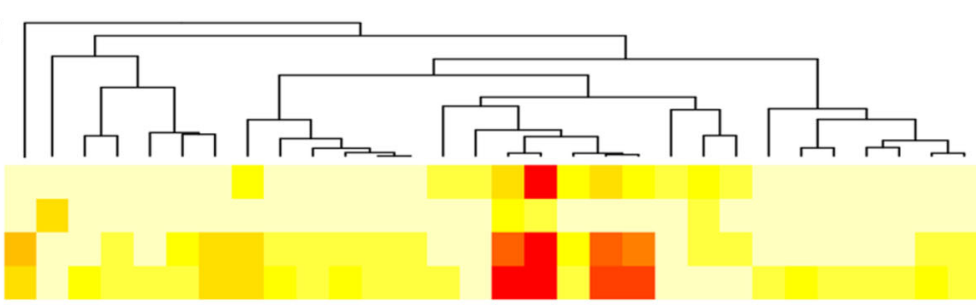

miR-499a-5p

miR-499b-5p

miR-499b-3p

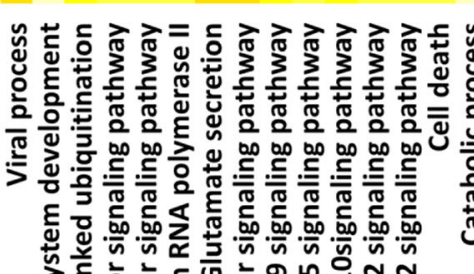

会至 高

突高

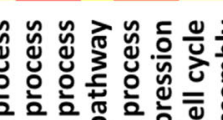

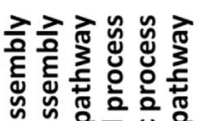

miR-499a-3p

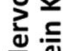

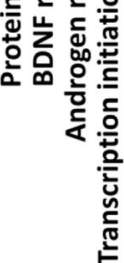
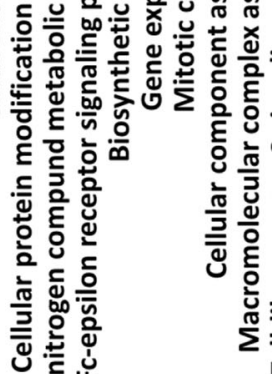

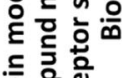

흥 잉

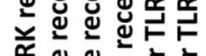

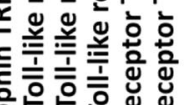

产辛

竞要

b

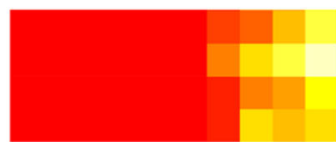

miR-499a-3p miR-499a-5p miR-499b-3p miR-499b-5p

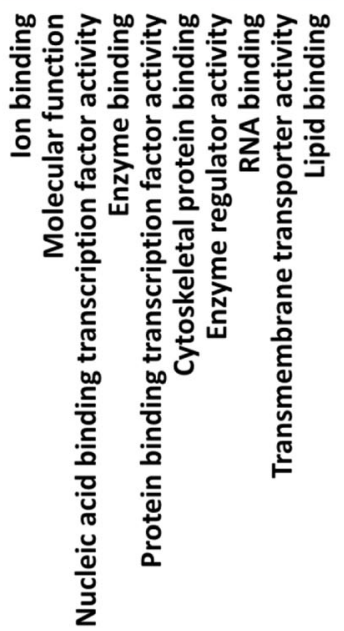

C

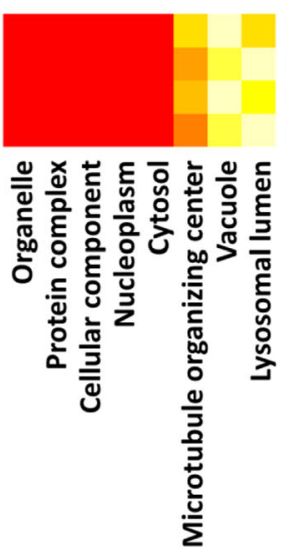

miR-499a-3p miR-499a-5p miR-499b-3p miR-499b-5p
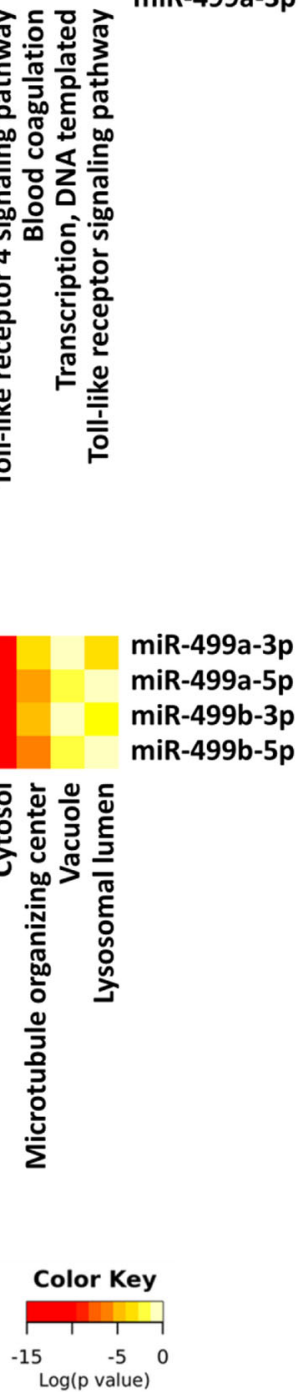

Fig. 5 Functional annotation clustering of target genes of MIR-499 gene family. Enrichment pathway analysis. Heat map showing a) Biological process, (b) Molecular functions and (c) Cellular components for mature forms of both hsa-miR-499a and hsa-miR-499b with $p$-values $<0.05$ and microT-CDS threshold 0.5. Degree of color is based on the significant $p$ values of the predicted algorithm by DIANA tools; the red has the highest score of statistical significance. The most pathogenomic feature shown in the heat maps is the involvement of miR-499 in different Toll-like receptor and Fc-epsilon receptor signaling pathways. Subsequent activation of these receptors release cytokines and mediators that drive immediate allergic airway reaction and contribute to intense eosinophil and lymphocyte infiltration in the airways 
(c. $2103+138 \mathrm{~A}>\mathrm{G}$ in ENST00000262873 transcript or c. $2100+138 \mathrm{~A}>\mathrm{G}$ in ENST00000618182 transcript). In addition, it represents the nucleotide number 73 (out of 122) of MIR499A on the forward strand (n.73A > G) within the sequence of miR-499a-3p and the nucleotide number 25 (out of 73) of MIR499B on the reverse strand (n.25 $\mathrm{T}>\mathrm{C}$ ) within the sequence of miR-499b-5p (Fig. 2d). Analyzing the secondary structure of pre-miR499a hairpin loop with rs3746444 SNP (either A or G alleles) via RNAfold and KineFold web-servers showed no effect of the alleles on the folding pattern. The SNP does not overlap any regulatory region or motif features. However, being in the seed region at the $5^{\prime}$ end of miR$499 a-3 p$ generates the possibility of creating an altered target gene set for that particular. Using PolymiRTS Database 3.0 platform, identified the disruption of 667 (41.8\%) genes of the miR-499a targets and creation of new 763 genes when A allele is substituted by $G$ at the seed region sequence: AAC[A/G]UCACAGCAAGUCU GUGCU. Comparing the two gene sets of each allele by miRmut2Go web-server revealed low functional similarity scores for the three GO domains; 0.378 for biological process, 0.401 for molecular function, and 0.528 for cellular component similarity scores, (Fig. 6). Further enrichment analysis of the new gene list targeted by $G$ allele was significantly involved in two KEGG pathways (glycan degradation and glycolysis/gluconeogenesis; $p=0.016$ and 0.031 respectively), 45 genes of them were significantly located on a single chromosome $(p=0.044)$.

\section{Genotyping of rs3746444 polymorphism in the study groups}

Baseline characteristics of patients and control groups in both discovery and replication stages are illustrated in Additional file 1: Table S1. Genotype distribution among the studied groups was in agreement with Hardy Weinberg equilibrium $(p>0.05)$. Analysis of the whole study population (211 patients and 300 controls) revealed that the minor allele frequency (MAF; G allele) in the control group was 0.34; the same allele was doubled in asthmatic cohort, accounting for $0.63(p<0.001)$. Correspondingly, there was higher frequency of GG genotype among asthmatic patients $(p<0.001)$, Table 1 . Both discovery and replication cohorts demonstrated similar trends in genotype and allele frequencies. Genetic association model analysis showed that individuals with $G$ variant were more likely to develop asthma than non-carriers under all association models ( $G$ versus $A$ : $\mathrm{OR}=3.27,95 \% \mathrm{CI}=2.53-4.22$; GG versus $\mathrm{AA}: \mathrm{OR}=9.52$, 95\% $\mathrm{CI}=5.61-16.5 ; \mathrm{AG}$ versus $\mathrm{AA}: \mathrm{OR}=2.13,95 \%$ $\mathrm{CI}=1.24-3.46 ; \mathrm{GG}+\mathrm{AG}$ versus $\mathrm{AA}: \mathrm{OR}=4.43,95 \%$ $\mathrm{CI}=2.88-6.82)$, Table 1 . High significant proportions of AG and GG genotypes were also observed in asthmatic children and adolescents of both gender $(p<0.001)$ (Fig. 7).
Genotype distribution according to disease characteristics of patients is shown in Table 2. There was no significant association of MIR-499a genotypes with any clinical or laboratory characteristics in the overall and stratified analysis. However, children and adolescents with early age at onset of asthma disease had higher frequency of GG genotype $(p=0.036)$. Additionally, GG homozygote patients have the lowest pre-bronchodilator $\mathrm{FEV}_{1}(p=0.047)$ and the worst bronchodilator response after Salbutamol inhalation represented in low peaked expiratory flow rate (PEFR) $(p=0.035)$ (Fig. 8). Similarly, data exploration by multivariate analysis did not demonstrate clustering of asthmatic patients according to their genotypes or any other clinical variables (Additional file 2: Figure S1).

\section{Discussion}

MicroRNAs are involved in various biological cellular processes. Aberrant transcriptomic signatures of various miRNAs have been detected in the airways and circulation of asthmatic patients [33]. Sequence variations within miRNA genes, especially in the mature miRNA seed region, may have a profound impact on miRNA biogenesis and function [34, 35]. In 2009, an inflammation-related MIR-499A was identified within the intronic sequence of the myosin $M Y H 7 B$ gene [36]. Several lines of evidence suggest that miR-499a play critical roles in orchestrating the immune response in various human disorders [11, 12, 37]. Bioinformatics analysis determined complement base pairing with important cytokines as IL-13 and IL-23, both are recognized effectors in asthma pathway. IL-13 signaling results in mucin hypersecretion, airway remodeling and fibrosis, and bronchial hyper-responsiveness [38]. IL-23 induction regulates allergic asthma through modulation of TH2 cell differentiation and eosinophilic infiltration [39]. Functional enrichment clustering illustrated the involvement of miR-499a-3a and miR-499a-3b in Toll-like receptor (TLC) signaling pathways that are implicated in the initial phase of the host defense against invading pathogens and allergens leading to $\mathrm{TH} 2$ activation [40, 41]. In addition, miR-499a and miR-499b are involved in Fcepsilon signaling pathway; cross-linking of IgE with FceRI receptors on mast cells, causes the release of leukotrienes and histamines essential for immediate allergic reaction. Other biological processes as gene expression, cellular protein modification process, transcription initiation from RNA polymerase II, and cellular component assembly are significantly mediated by miR-499 gene family.

Using various computational tools, miR-499a and miR499b were predicted to target key molecules in asthma-related KEGG pathways. They could modulate 18 targets in mucin type O-biosynthesis pathway related to polypeptide $\mathrm{N}$-Acetylgalactosaminyltransferase (GALNT) 


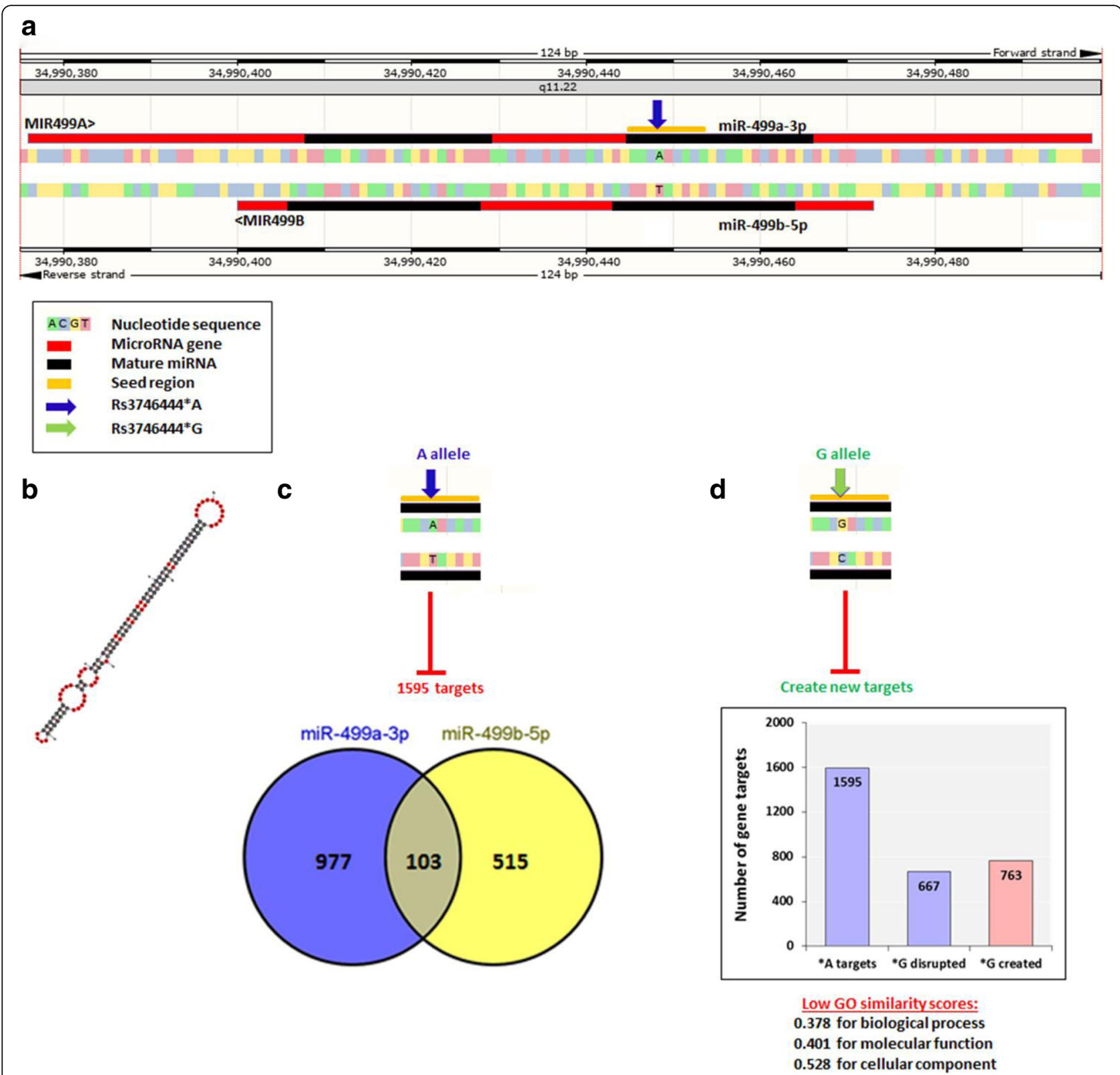

Fig. 6 Structural and functional impact of rs3746444 variant in MIR-499A gene. a) Genomic location of the SNP on both strands. It represents the nucleotide number 73 (out of 122) of MIR499A on the forward strand (n.73A > G) within the sequence of miR-499a-3p and the nucleotide number 25 (out of 73) of MIR499B on the reverse strand (n.25 T > C) within the sequence of miR-499b-5p. (b) Predicted folding pattern of precursor stem loop structure by RNAfold and KineFold web-servers. No effect of the alleles on the folding pattern was detected. (c) Targets of A allele in miR-499a-3p and miR-499b-5p. (d) Predicted functional effects of $\mathrm{G}$ allele on gene targets and gene ontology. Being in the seed region at the 5' end of miR-499a-3p generates the possibility of creating an altered target gene set for that particular. Using PolymiRTS Database 3.0 platform, identified the disruption of 667 (41.8\%) genes of the miR-499a targets and creation of new 763 genes when A allele is substituted by $G$ at the seed region sequence: AAC[A/G]UCACAGCAAGUCUGUGCU

genes. Hyperglycosylated mucins are commonly found in chronic inflammatory airway diseases such as asthma, for which inhibition of mucin and their glycosylation could contribute in controlling the disease [42]. The next most significant pathway was fatty acid biosynthesis with 5 target genes. Recent studies demonstrated the vital role of fatty acids in the formation of inflammatory mediators relevant to the pathophysiology of asthma [43]. Another identified pathway that is highly impacted by miR-499 gene family is glycosphingolipid (GSL) biosynthesis. $\beta$ Glycosphingolipids have emerged as a family of potential ligands for natural killer (NK) cells. Extensive infiltration of NK cells in the airway bronchial mucosa is considered one of the prominent driver in asthma development via 


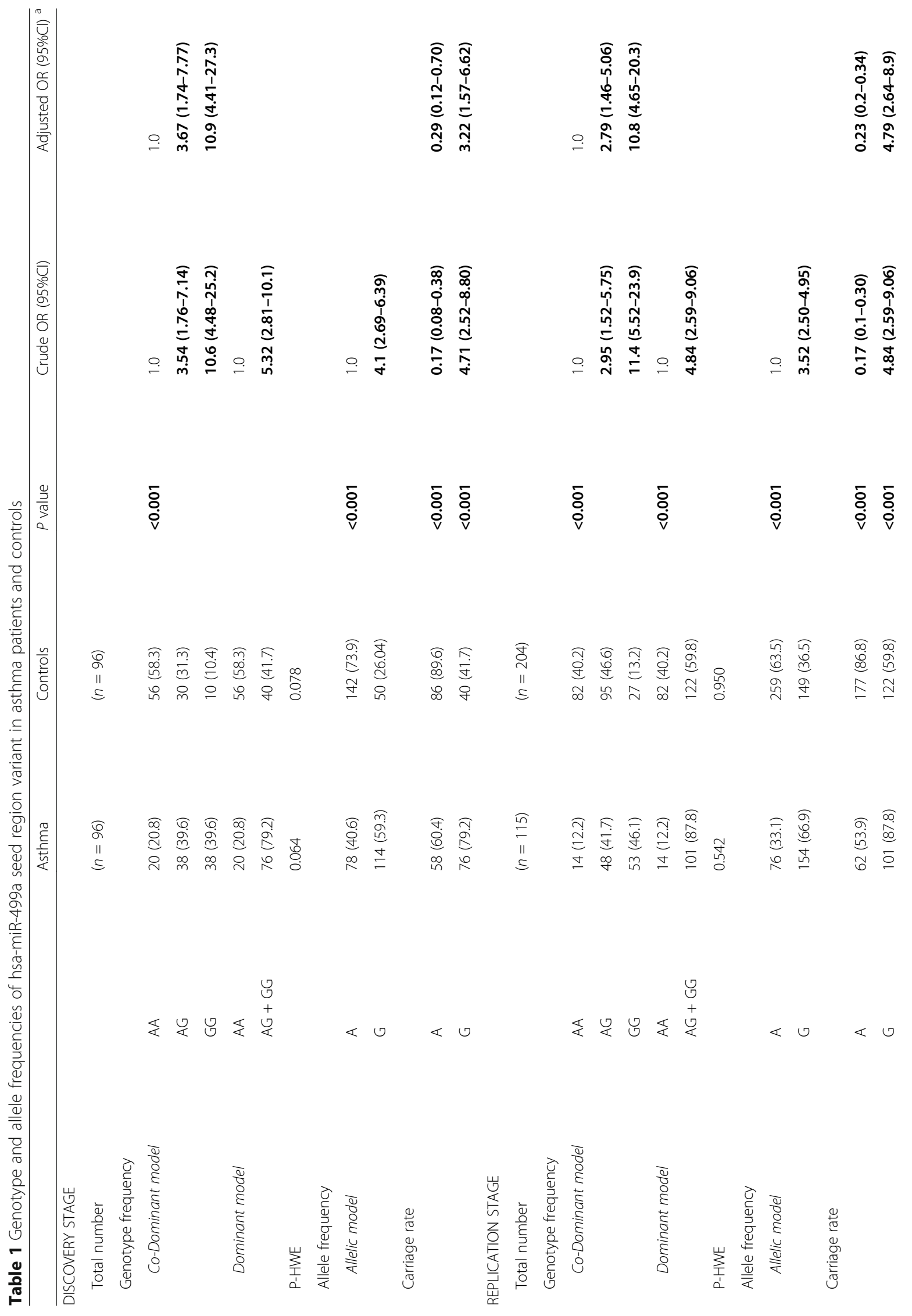




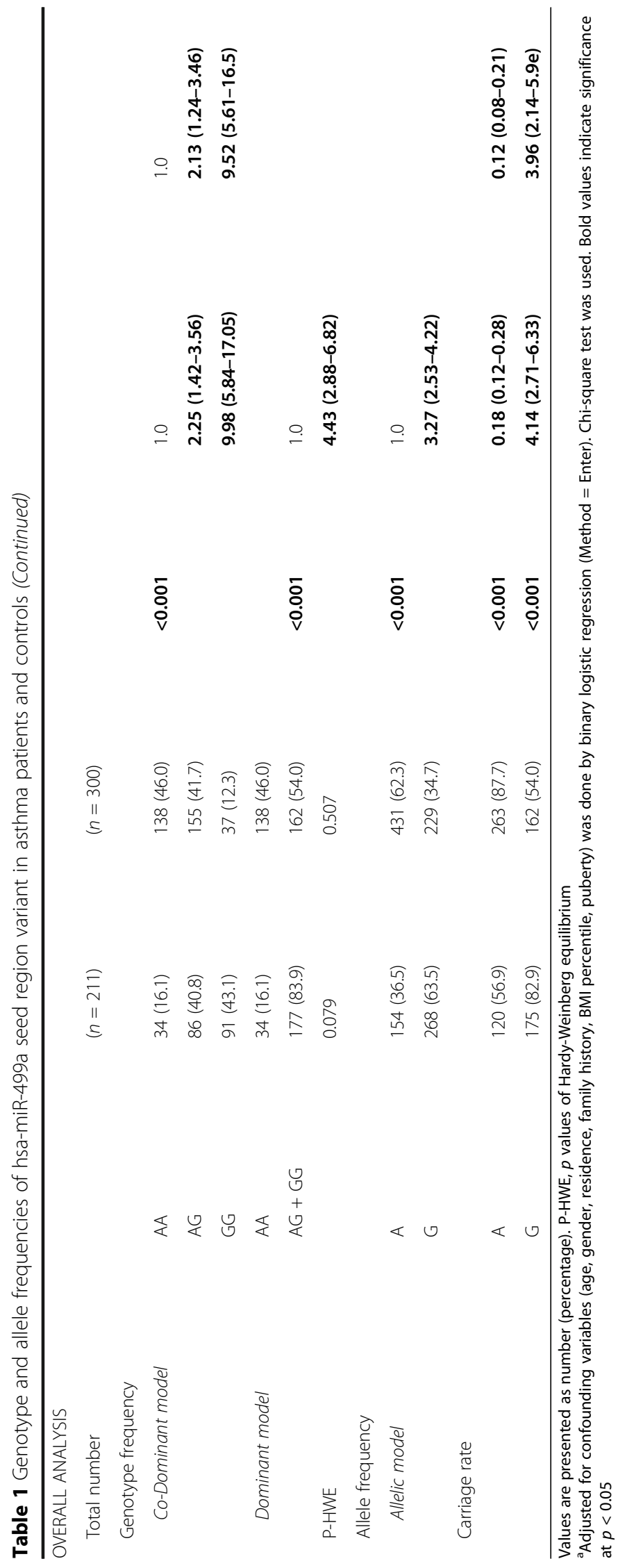




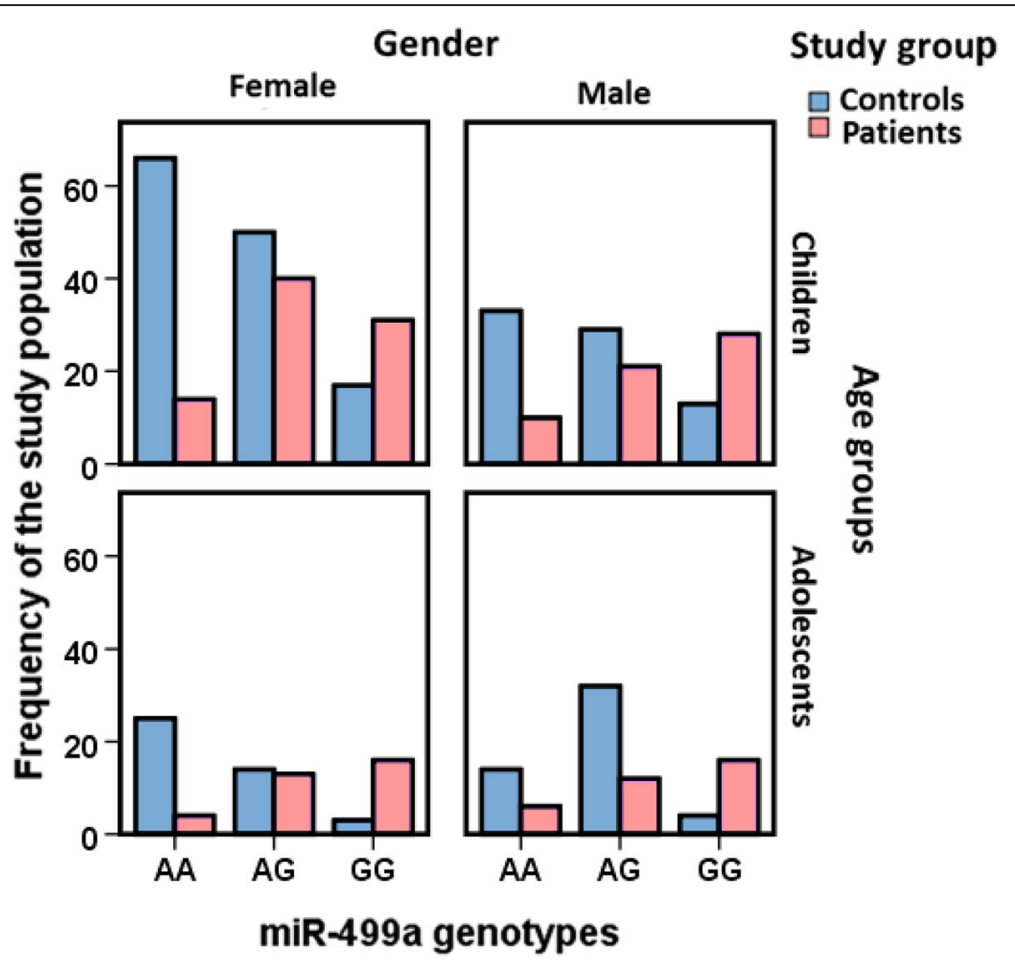

Fig. 7 Allelic discrimination analysis of rs3746444 MIR-499A polymorphism in the study population. Genotype frequencies in patients and controls stratified by age and gender. AA genotype was more frequent in controls than their counterparts. Chi square test was used. Statistical significance at $p<0.05$

inducing the secretion of $\mathrm{TH} 2$ cytokines that results in airway hyper-responsiveness and inflammation [44]. Lowering GSL levels in mast cells in an animal model of asthma was found to ameliorate disease manifestations [45]. Both mature forms of miR-499a and miR-499b were predicted to target about 40 and 60 genes in TGF-beta and TNF signaling pathways, important mediators contributing in lung tissue fibrosis and proliferation of smooth muscle cells with subsequent airway remodeling [46]. We also identified several targets for miR-499 gene family in adherence junction and focal adhesion pathways. Tight junctions not only seal the pulmonary epithelium and maintain the structural integrity of the airway walls, but also act as key regulators for epithelial cell proliferation and differentiation. Dysfunction of this homeostasis could participate in airway wall remodeling in asthma disease [47].

Within MIR499A and MIR499B genes, bioinformatics analysis revealed the presence of a common polymorphism at the position 20:34,990,448 within the seed region of mature miR-499a-3p (rs3746444; AC[A/G]UCAC). In the current study, we genotyped rs3746444 polymorphism in asthmatic children and adolescents compared to controls. Genotype frequencies did not deviate from the Hardy-Weinberg equilibrium in the study groups. G variant was associated with increased susceptibility to develop bronchial asthma under all genetic association models. Despite the MAF (G) represented 0.26 in the control group, it accounted for 0.59 among patients. The same allele was found to be associated with poor prebronchodilator $\mathrm{FEV}_{1}$ in asthmatic patients. In addition, homozygotes for $\mathrm{G}$ had the worst bronchodilator response after Salbutamol inhalation. That was consistent with prior studies; $G$ allele was also associated with increased risk of developing of several autoimmune and inflammatory diseases. miR-499a"G carriers showed higher susceptibility to RA in Egyptians [12] and Iranian people [48], Behcet's disease in Turks [15], ulcerative colitis in Japanese [49], ankylosing spondylitis [16], idiopathic recurrent spontaneous abortion in Koreans [50], and coronary artery disease $[51,52]$. In addition, individuals with rs3746444*G allele showed a more active phenotype of RA in Egyptian female patients [53] and a severe course of multiple sclerosis [54].

Though our in silico data analysis revealed that the substitution of A > G has no dramatic effect on the folding pattern of the hairpin loop structure, each allele was predicted to have different set of gene targets. A total of 1890 genes is influenced by mature miR-499a with rs3746444*A variant. In contrast, rs3746444*G allele could delete $41.8 \%$ of these genes and create new 763 targets. By conducting functional annotation clustering and enrichment analysis, our results illustrated that gene 


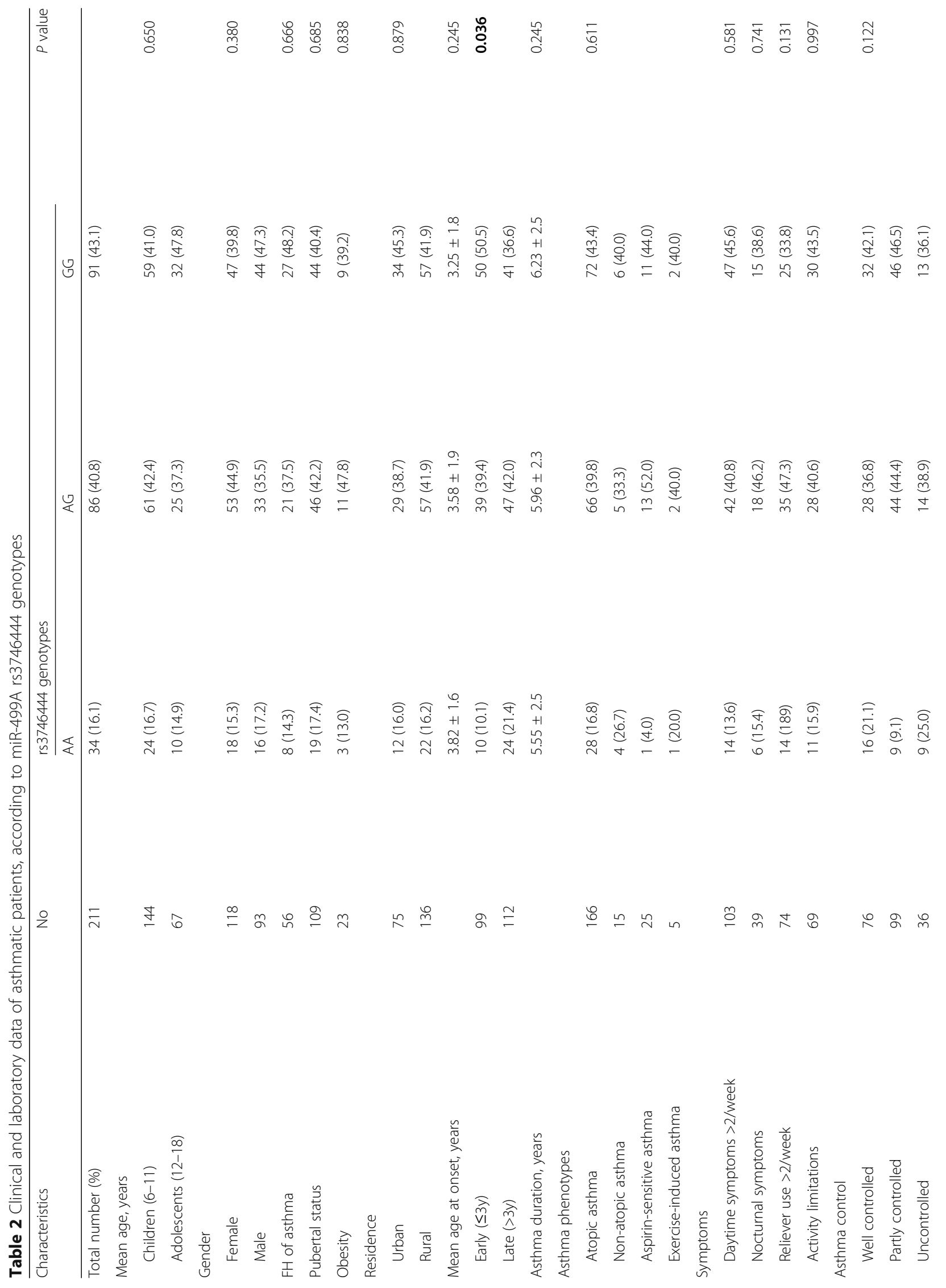


Toraih et al. Respiratory Research (2017) 18:169

Page 15 of 18

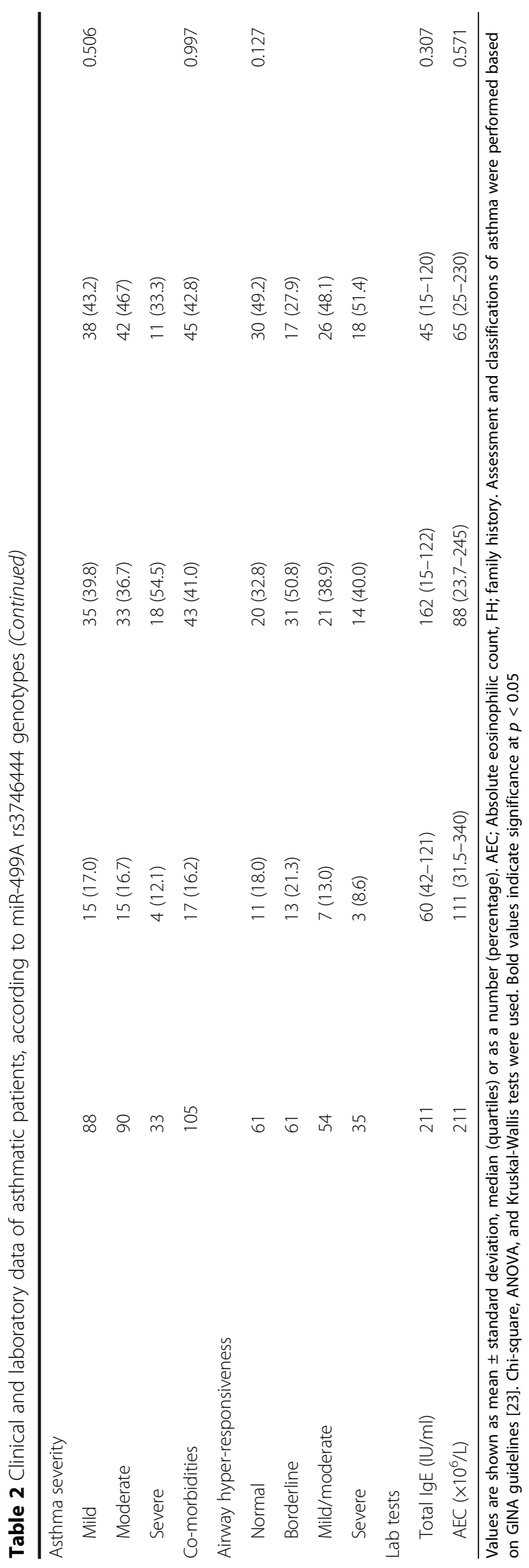




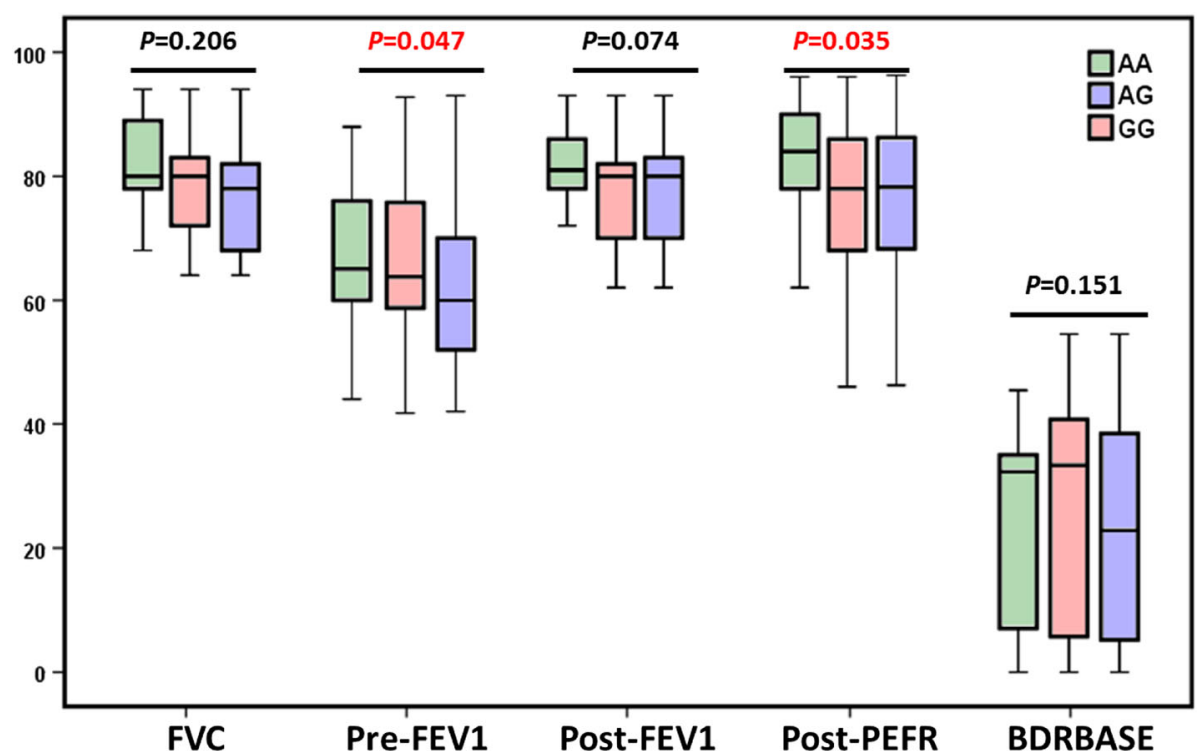

Fig. 8 Bronchodilator response in asthmatic patients, according to MIR-499a genotypes. Pre-FEV1: pre-bronchodilator forced expiratory volume in one second before salbutamol inhalation, post-FEV1: post-bronchodilator forced expiratory volume in one second after salbutamol inhalation, post-PEFR: post-peaked expiratory flow rate, BDRBASE: change in FEV1 as a percent of baseline FEV1 [BDRBASE $=(($ postbronchodilator FEV1 prebronchodilator FEV1) / prebronchodilator FEV1) $\times$ 100]

sets for each allele only shared the same $37.8 \%, 40.1 \%$, and $52.8 \%$ for biological process, molecular function, and cellular components, respectively. In particular, G allele targets were significantly involved in two new KEGG pathways (other glycan degradation and glycolysis/gluconeogenesis). In the former pathway, miR-499 can target both aspartylglucosaminidase ( $A G A$ ) gene involved in the catabolism of $\mathrm{N}$-linked oligosaccharides of glycoproteins in the lysosomes, and galactosidase beta 1 (GLB1) gene, which plays functional roles in the formation of extracellular elastic fibers and in the development of connective tissue. In the other pathway for glycolysis/gluconeogenesis, miR-499 mainly target aldehyde dehydrogenase 1 family member A3 (ALDH1A3) and alcohol dehydrogenase 1 beta polypeptide $(A D H 1 B)$ genes, members of the enzyme family which metabolize a wide variety of substrates as multiple inositol-polyphosphate phosphatase 1, an important second messenger in eukaryotic cells. $A L D H 1 A 3$ gene was found to be over-expressed more than two times in asthmatic patients and 3.5 times during exacerbations [55]. Further, in vivo functional analysis is warranted to explore the mechanistic regulation of these targets in the etiopathogenesis of asthma.

In some previous studies, computational methods were devised to study the effects of air-borne dust through high fidelity computational simulation of different particle sizes over several breathing cycles [56], modeling lung pressures [57], and developing 3D
Euler Lagrangian models to obtain the regional deposition of the poly-dispersed drug Budesonide [58]. It would be a beneficial next step to assess the pharmacogenomics effect of variants with the regional deposition of dust particles and drug delivery to the respiratory tract during respiration.

To the best of our knowledge, this is the first study highlighting the role of miR-499 rs3746444 in bronchial asthma disease. However, some limitations need to be addressed. First, the small relative sample size in the current study might underestimate the synergistic effect of miR-499 SNP with environmental exposure. Second, the impact of the SNP was not correlated with the miRNA expression profile. Third, functional assessment of targets involved in bronchodilator response and methacholine challenge test is mandatory to develop more efficient therapeutic strategies.

\section{Conclusions}

In conclusion, our study suggested that the rs3746444 (A > G) polymorphism in miR-499 gene family might contribute to the susceptibility of asthma in children and adolescents with bronchial asthma. Additional studies, including larger cohorts with diverse ethnic background, and functional tests are warranted to explore the immunomodulatory mechanism of miR-499 genes in bronchial asthma for developing more specific theranostic agents. 


\section{Additional files}

Additional file 1: Table S1. Baseline characteristics of the study groups. Table S2. Predicted target gene sets for mature miR-499a and miR-499b. (http://www.microrna.gr/microT-CDS). (ZIP 79 kb)

Additional file 2: Figure S1. Principal components analysis of asthmatic patients. Ordination plot constructed using 211 patients strand and 20 clinical and laboratory variables. Samples are distributed along two axes. Axis 1 explains 22.6\% of variance among patients, whereas axis 2 resolves $11.3 \%$ of variance. Samples are scattered and colored according to their genotype; AA (red), AG (green), and GG (blue). PCA did not reveal clustering of patients according to their genotypes. (DOCX $95 \mathrm{~kb}$ )

\section{Acknowledgements}

The authors thank the Oncology Diagnostic Unit and the Center of Excellence in Molecular and Cellular Medicine, Suez Canal University, Ismailia, Egypt for providing the facilities for performing the research work as well as we thank all participants who agree to participate in the current study.

\section{Funding}

No sources of funding were used for this work.

\section{Availability of data and materials}

All data generated or analyzed during this study are included in this published article and its supplementary information files.

\section{Authors' contributions}

EAT, EA, NMB, GMH, MSF conceived and designed the experiments, MHH. ER and $\mathrm{NMB}$ recruited the study samples and clinical patient data, EAT, $M H \mathrm{H}$, $E R, N M B$ and MSF contributed parts of the experiments, EAT contributed to the bioinformatic and the statistical analyses. All authors contributed the reagents and materials needed for the current work, and all authors contributed in writing, reading and approval of the final manuscript.

\section{Ethics approval and consent to participate}

The study was conducted in accordance with the guidelines in the Declaration of Helsinki and it has been approved by the Medical Research Ethics Committee of Faculty of Medicine, Suez Canal University. Written informed consent was obtained from all participants.

\section{Consent for publication}

Not applicable.

\section{Competing interests}

The authors declare that they have no competing interests.

\section{Publisher's Note}

Springer Nature remains neutral with regard to jurisdictional claims in published maps and institutional affiliations.

\footnotetext{
Author details

${ }^{1}$ Genetics Unit, Department of Histology and Cell Biology, Faculty of Medicine, Suez Canal University, Ismailia P.O. 41522, Egypt. Pulmonologist, Ministry of Health, Cairo, Egypt. ${ }^{3}$ Department of Clinical Biochemistry (Medical Genetics), Faculty of Medicine, Jazan University, Jazan, Saudi Arabia. ${ }^{4}$ Department of Chest Diseases and Tuberculosis, Faculty of Medicine, Suez Canal University, Ismailia P.O. 41522, Egypt. ${ }^{5}$ Department of Pediatrics, Faculty of Medicine, Suez Canal University, Ismailia, Egypt. ${ }^{6}$ Department of Medical Biochemistry, Faculty of Medicine, Mansoura University, Mansoura, Egypt. 'Department of Medical Biochemistry, Faculty of Medicine, Suez Canal University, Ismailia P.O. 41522, Egypt. ${ }^{8}$ Department of Medical Biochemistry, Faculty of Medicine, Northern Border University, Arar, Kingdom of Saudi Arabia.
}

Received: 15 May 2017 Accepted: 25 August 2017

Published online: 08 September 2017

\section{References}

1. Li S, Xie X, Song Y, Jiang H, Wu X, Su X, Yang L, Li M. Association of TLR4 (896A/G and 1196C/T) gene polymorphisms with asthma risk: a metaanalysis. Med Sci Monit. 2015;21:3591-9.

2. Hussein MH, Toraih EA, Aly NM, Riad E, Fawzy MS. A passenger strand variant in miR-196a2 contributes to asthma severity in children and adolescents: a preliminary study. Biochem Cell Biol. 2016;94:347-57.

3. Tang DD. Critical role of actin-associated proteins in smooth muscle contraction, cell proliferation, airway hyperresponsiveness and airway remodeling. Respir Res. 2015;16:134.

4. Nakajima H, Hirose K. Role of IL-23 and Th17 cells in airway inflammation in asthma. Immune Netw. 2010;10:1-4.

5. Hall SC, Agrawal DK. Vitamin D and bronchial asthma: an overview of data from the past 5 years. Clin Ther. 2017

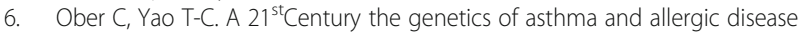
perspective. Immunol Rev. 2011;242:10-30.

7. Ardekani AM, Naeini MM. The role of MicroRNAs in human diseases. Avicenna Journal of Medical Biotechnology. 2010;2:161-79.

8. Toraih EA, Mohammed EA, Farrag S, Ramsis N, Hosny S. Pilot study of serum MicroRNA-21 as a diagnostic and prognostic biomarker in Egyptian breast cancer patients. Mol Diagn Ther. 2015:19:179-90.

9. Fawzy MS, Hussein MH, Abdelaziz EZ, Yamany HA, Ismail HM, Toraih EA. Association of MicroRNA-196a2 Variant with Response to Short-Acting beta 2-Agonist in COPD: An Egyptian Pilot Study. PLoS One. 2016;11:2.

10. Esteller M. Non-coding RNAs in human disease. Nat Rev Genet. 2011;12: 861-74

11. Ji Q, Jiang $Q$, Yan W, Li X, Zhang Y, Meng P, Shao M, Chen L, Zhu H, Tian N. Expression of circulating microRNAs in patients with ST segment elevation acute myocardial infarction. Minerva Cardioangiol. 2015;63:397-402.

12. Toraih EA, Ismail NM, Toraih AA, Hussein MH, Fawzy MS. Precursor miR-499a variant but not miR-196a2 is associated with rheumatoid arthritis susceptibility in an Egyptian population. Mol Diagn Ther. 2016:20:279-95.

13. Song GG, Bae SC, Seo YH, Kim JH, Choi SJ, Ji JD, Lee YH. The association between susceptibility to inflammatory arthritis and miR-146a, miR-499 and IRAK1 polymorphisms. A meta-analysis. Z Rheumatol. 2015;74:637-45.

14. Chen W, Shao D, Gu H, Gong J, Zhang J. Hsa-mir-499 rs3746444 T/C polymorphism is associated with increased risk of coronary artery disease in a Chinese population. Acta Cardiol Sin. 2017;33:34-40.

15. Oner T, Yenmis G, Tombulturk K, Cam C, Kucuk OS, Yakicier MC, Dizman D, Sultuybek GK. Association of pre-miRNA-499 rs3746444 and pre-miRNA-146a rs2910164 polymorphisms and susceptibility to Behcet's disease. Genet Test Mol Biomarkers. 2015;19:424-30.

16. Xu HY, Wang ZY, Chen JF, Wang TY, Wang LL, Tang LL, Lin XY, Zhang CW, Chen BC. Association between ankylosing spondylitis and the miR-146a and miR-499 polymorphisms. PLoS One. 2015:10:e0122055.

17. Wilm A, Higgins DG, Notredame C. R-coffee: a method for multiple alignment of non-coding RNA. Nucleic Acids Res. 2008:36:e52.

18. Bhattacharya A, Cui Y. miR2GO: comparative functional analysis for microRNAs. Bioinformatics. 2015:31:2403-5.

19. Vlachos IS, Zagganas K, Paraskevopoulou MD, Georgakilas G, Karagkouni D, Vergoulis T, Dalamagas T, Hatzigeorgiou AG. DIANA-miRPath v3.0: deciphering microRNA function with experimental support. Nucleic Acids Res. 2015;43:W460-6.

20. Bhattacharya A, Ziebarth JD, Cui Y. PolymiRTS database 3.0: linking polymorphisms in microRNAs and their target sites with human diseases and biological pathways. Nucleic Acids Res. 2014:42:D86-91.

21. Bruno AE, Li L, Kalabus JL, Pan Y, Yu A, Hu Z. miRdSNP: a database of disease-associated SNPs and microRNA target sites on 3'UTRs of human genes. BMC Genomics. 2012;13:44.

22. Keller A, Backes C, Al-Awadhi M, Gerasch A, Küntzer J, Kohlbacher O Kaufmann M, Lenhof HP. GeneTrailExpress: a web-based pipeline for the statistical evaluation of microarray experiments. BMC Bioinformatics. 2008:9:552.

23. Global Initiative for Asthma. Global Strategy for Asthma Management and Prevention. Revised 2014 Vancouver, GINA, 2014. Available from: www. ginasthma.org [Accessed 16 December 2016].

24. Fawzy MS, Alhadramy O, Hussein MH, Ismail HM, Ismail NM, Biomy NM, Toraih EA. Functional and structural impact of ATP-binding cassette 
transporter A1 R219K and 1883M gene polymorphisms in obese children and adolescents. Mol Diagn Ther. 2015;19:221-34.

25. Marshall WA, Tanner JM. Variations in pattern of pubertal changes in boys and girls. Arch Dis Child. 1969;44:291-303.

26. Soriano JB, Alfageme I, Almagro P, Casanova C, Esteban C, Soler-Cataluña JJ, de Torres JP, Martinez-Camblor P, Miravitlles M, Celli BR, Marin JM. Distribution and prognostic validity of the new global initiative for chronic obstructive lung disease grading classification. Chest. 2013;143:694-702.

27. Hussein MH, Sobhy KE, Sabry IM, El Serafi AT, Toraih EA. Beta2-adrenergic receptor gene haplotypes and bronchodilator response in Egyptian patients with chronic obstructive pulmonary disease. Adv Med Sci. 2017;62:193-201.

28. Crapo RO, Casaburi R, Coates AL, Enright PL, Hankinson JL, Irvin CG, Maclntyre NR, McKay RT, Wanger JS, Anderson SD, Cockcroft DW, Fish JE, Sterk PJ. Guidelines for methacholine and exercise challenge testing-1999. This official statement of the American Thoracic Society was adopted by the ATS Board of directors, July 1999. Am J Respir Crit Care Med. 2000;161 309-29.

29. Pellegrino R, Viegi G, Brusasco V, Crapo RO, Burgos F, Casaburi R, Coates A, van der Grinten CPM, Gustafsson P, Hankinson J, et al. Interpretative strategies for lung function tests. Eur Respir J. 2005;26:948-68.

30. Demirjian M, Rumbyrt JS, Gowda VC, Klaustermeyer WB. Serum IgE and eosinophil count in allergic rhinitis-analysis using a modified Bayes' theorem. Allergol Immunopathol (Madr). 2012;40:281-7.

31. Toraih E, Hussein MH, Badran DI. Beta2-adrenergic receptor gene polymorphisms in Egyptian patients with acute myocardial infarction. Advances in Molecular Biology. 2014;2014:471635.

32. Toraih EA, Fawzy MS, El-Falouji Al, Hamed EO, Nemr NA, Hussein MH, Abd el Fadeal NM. Stemness-related transcriptional factors and homing gene expression profiles in hepatic differentiation and cancer. Mol Med. 2016;22

33. Fang C, Lu W, Li C, Peng X, Wang Y, Huang X, Yao Z, Cai N, Huang Y, Zhang $X$, Tan J. MiR-3162-3p is a novel MicroRNA that exacerbates asthma by regulating $\beta$-catenin. PLoS One. 2016;11:e0149257.

34. Sun G, Yan J, Noltner K, Feng J, Li H, Sarkis DA, Sommer SS, Rossi JJ. SNPs in human miRNA genes affect biogenesis and function. RNA. 2009;15:1640-51.

35. Toraih EA, Fawz MS, Elgazzaz MG, Hussein MH, Shehata RH, Daoud HG. Combined genotype analyses of precursor miRNA196a2 and 499a variants with hepatic and renal cancer susceptibility a preliminary study. Asian Pac J Cancer Prev. 2016;17:3369-75.

36. van Rooij E, Quiat D, Johnson BA, Sutherland LB, Qi X, Richardson JA, Kelm RJ, Olson EN. A family of microRNAs encoded by myosin genes governs myosin expression and muscle performance. Dev Cell. 2009;17:662-73.

37. Liu J, Liang X, Zhou D, Lai L, Xiao L, Liu L, Fu T, Kong Y, Zhou Q, Vega RB, et al. Coupling of mitochondrial function and skeletal muscle fiber type by a miR-499/Fnip1/AMPK circuit. EMBO Mol Med. 2016;8:1212-28.

38. Rael EL, Lockey RF. Interleukin-13 signaling and its role in asthma. World Allergy Organ J. 2011:4:54-64.

39. Peng J, Yang XO, Chang SH, Yang J, Dong C. IL-23 signaling enhances Th2 polarization and regulates allergic airway inflammation. Cell Res. 2010;20:62-71.

40. Liew FY, Patel M, Xu D: Toll-like receptor 2 signalling and inflammation. Ann Rheum Dis 2005, 64 Suppl 4:iv104-105.

41. Oglesby IK, McElvaney NG, Greene CM. MicroRNAs in inflammatory lung disease-master regulators or target practice? Respir Res. 2010;11:148.

42. Pedersen JW, Bennett EP, Schjoldager KT, Meldal M, Holmér AP, Blixt O, Cló E, Levery SB, Clausen H, Wandall HH. Lectin domains of polypeptide GalNAC transferases exhibit glycopeptide binding specificity. J Biol Chem. 2011;286: 32684-96.

43. Wendell SG, Baffi C, Holguin F. Fatty acids, inflammation, and asthma. J Allergy Clin Immunol. 2014;133:1255-64.

44. Adar T. Ilan Y: beta-glycosphingolipids as immune modulators. J Immunotoxicol. 2008;5:209-20.

45. Karman J, Tedstone JL, Gumlaw NK, Zhu Y, Yew N, Siegel C, Guo S, Siwkowski A, Ruzek M, Jiang C, Cheng SH. Reducing glycosphingolipid biosynthesis in airway cells partially ameliorates disease manifestations in a mouse model of asthma. Int Immunol. 2010;22:593-603.

46. Szymczak I, Wieczfinska J, Pawliczak R. Molecular background of miRNA role in asthma and COPD: an updated insight. Biomed Res Int. 2016;2016: 7802521.

47. Davies DE. Epithelial barrier function and immunity in asthma. Ann Am Thorac Soc. 2014;11(Suppl 5):S244-51.

48. Hashemi M, Eskandari-Nasab E, Zakeri Z, Atabaki M, Bahari G, Jahantigh M, Taheri M, Ghavami S. Association of pre-miRNA-146a rs2910164 and pre-
miRNA-499 rs3746444 polymorphisms and susceptibility to rheumatoid arthritis. Mol Med Rep. 2013;7:287-91.

49. Okubo M, Tahara T, Shibata T, Yamashita H, Nakamura M, Yoshioka D, Yonemura J, Kamiya Y, Ishizuka T, Nakagawa Y, et al. Association study of common genetic variants in pre-microRNAs in patients with ulcerative colitis. J Clin Immunol. 2011;31:69-73.

50. Jeon YJ, Choi YS, Rah H, Kim SY, Choi DH, Cha SH, Shin JE, Shim SH, Lee WS, Kim NK. Association study of microRNA polymorphisms with risk of idiopathic recurrent spontaneous abortion in Korean women. Gene. 2012; 494:168-73.

51. Zhi H, Wang L, Ma G, Ye X, Yu X, Zhu Y, Zhang Y, Zhang J, Wang B. Polymorphisms of miRNAs genes are associated with the risk and prognosis of coronary artery disease. Clin Res Cardiol. 2012;101:289-96.

52. Fawzy MS, Toraih Eman A, Hamed EO, Hussein MH, Ismail HM. Association of MIR-499a expression and seed region variant (rs3746444) with cardiovascular disease in Egyptian patients. Acta Cardiol. 2017; https://doi. org/10.1080/00015385.2017.1351243.

53. El-Shal AS, Aly NM, Galil SM, Moustafa MA, Kandel WA. Association of microRNAs genes polymorphisms with rheumatoid arthritis in Egyptian female patients. Joint Bone Spine. 2013;80:626-31.

54. Kiselev I, Bashinskaya V, Kulakova O, Baulina N, Popova E, Boyko A, Favorova O. Variants of MicroRNA genes: gender-specific associations with multiple sclerosis risk and severity. Int J Mol Sci. 2015;16:20067-81.

55. Hershey $\mathrm{G}$, Aronow B: Altered gene expression profiles in stable versus acute childhood asthma. US7,919,240B2 2011. Available at https://www. google.com/patents/US7919240 (last accessed May 6th, 2017).

56. Kannan R, Guo P, Przekwas A. Particle transport in the human respiratory tract: formulation of a nodal inverse distance weighted Euler-Lagrangian transport and implementation of the wind-Kessel algorithm for an oral delivery. Int J Numer Method Biomed Eng. 2016;32

57. Kannan R, Chen ZJ, Singh N, Przekwas A, Delvadia R, Tian G, Walenga R. A quasi-3D wire approach to model pulmonary airflow in human airways. Int J Numer Method Biomed Eng. 2017;33

58. Ravi Kannan R, Przekwas AJ, Singh N, Delvadia R, Tian G, Walenga R. Pharmaceutical aerosols deposition patterns from a dry powder inhaler: Euler Lagrangian prediction and validation. Med Eng Phys. 2017:42:35-47.

\section{Submit your next manuscript to BioMed Central and we will help you at every step:}

- We accept pre-submission inquiries

- Our selector tool helps you to find the most relevant journal

- We provide round the clock customer support

- Convenient online submission

- Thorough peer review

- Inclusion in PubMed and all major indexing services

- Maximum visibility for your research

Submit your manuscript at www.biomedcentral.com/submit
Biomed Central 